\title{
Pseudocisto de pâncreas associado a derrame pleural maciço*
}

\author{
evandro Monteiro S. de Magalhães ${ }^{1}$, J oaquim Carlos Ávila Terra², \\ luciana Borém Pimenta de Figueiredo ${ }^{3}$, Luís Henrique Serra Miranda ${ }^{3}$
}

Os autores relatam um achado de derrame pleural maciço decorrente de pseudocisto de pâncreas.

Para auxílio de diagnóstico foram realizadas radiografia, ultra-sonografia, REED, tomografia computadorizada toracoabdominal e dosagem de amilase no líquido pleural. 0 paciente foi submetido a tratamento clínico com suporte nutricional e toracocentese com esvaziamento pleural de $3.200 \mathrm{ml}$. A pós estabilização clínica/nutricional, foi indicada laparotomia, com a retirada do pseudocisto. 0 paciente apresentou boa evolução no pós-operatório, recebendo alta em boas condições clínicas. A partir do caso relatado, é feita uma revisão da literatura sobre a ocorrência de derrames pleurais associados à doença pancreática e seu diagnóstico. (J Pneumol 2002;28(3):159-162)

\section{Pancreatic pseudocyst associated to massive pleural effusion}

The authors report a case of pancreatic pseudocyst associated with massive pleural effusion. The patient was submitted to $X$ rays, REED, ultrasonography, thoracoabdominal computerized tomography and amylase dosing in the pleural liquid. Clinical treatment included dietary support and thoracocentesis with drainage of about $3,200 \mathrm{ml}$. Patient was submitted to laparotomy to remove the pseudocyst, and was discharged in good clinical conditions. Based on this report, the authors analyze and review the literature for the occurrence of pleural effusion associated with pancreatic diseases and diagnoses.

Descritores - Pseudocisto pancreático. Derrame pleural. Tomografia computadorizada por raios X. Laparotomia.

Key words - Pancreatic pseudocyst. Pleural effusion. Computerized X-ray tomography. Laparotomy.

\section{INTRODUÇÃO}

Pseudocisto $(\mathrm{PC})$ de pâncreas é uma coleção que contém tecidos, líquido, fragmentos, enzimas pancreáticas e sangue, encerrada por parede não epitelizada de tecido fibroso ou de granulação, que se origina de pancreatite aguda ou crônica ou de trauma pancreático. Aproximadamente, $5 \%$ dos pacientes com PC desenvolvem derra-

* Trabalho realizado na Faculdade de Ciências Médicas de Alfenas, MG (FCM-Unifenas).

1. Chefe do Departamento de Pneumologia.

2. Cirurgião Geral do Hospital IMESA e da Santa Casa de Misericórdia de Alfenas.

3. Acadêmico do curso de graduação.

Endereço para correspondência - Luís Henrique Serra Miranda, Alameda Márcio Paulino da Costa, 316 - 37130-000 - Alfenas, MG. Tel. (35) 3291-1495. E-mail: Ih_ique@yahoo.com.br.

Recebido para publicação em 3/12/01. Aprovado, após revisão, em 21/2/02.
Siglas e abreviaturas utilizadas neste trabalho

$P C$ - Pseudocisto

REED - Raio X de esôfago estômago duodeno

me pleural, sendo este mais freqüente à esquerda como decorrência de fístula pancreático-pleural. Aqui é descrito um caso incomum de PC de pâncreas associado a derrame pleural maciço devido à extensão do pseudocisto por trajeto direto com o espaço pleural passando pelo diafragma.

\section{Relato do CASO}

Homem de 39 anos, lavrador, natural e procedente de Poço Fundo, MG, procurou o serviço de Pneumologia apresentando tosse com expectoração mucopurulenta fétida havia cerca de três semanas; dispnéia aos médios esforços e emagrecimento de cerca de 5,0kg nos últimos dois meses, adinamia, inapetência, febre não mensurada. Era etilista (dois copos de aguardente/dia) havia cinco anos e tabagista (40 cigarros/dia) havia 15 anos.

0 exame físico revelou paciente em regular estado geral, pele descamativa, hipocorado $(+/ 4+)$, cavidade oral 


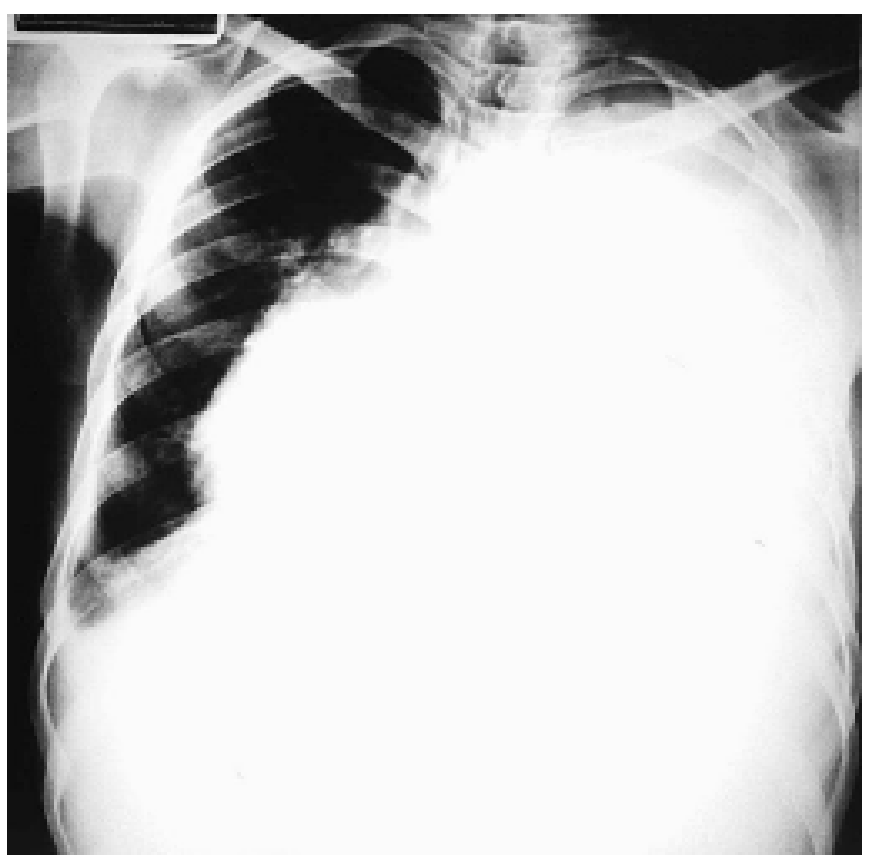

Figura 1 - Radiografia de tórax realizada uma semana após a internação

em péssimas condições (boca séptica). Sinais vitais: PA: 110x80mmHg, FR: 20irpm, FC: 110bpm.

Ao exame torácico, apresentava expansibilidade diminuída, com murmúrio vesicular abolido nos dois terços inferiores do hemitórax esquerdo e submacicez à percussão.

0 exame abdominal revelou, à palpação, abdome tenso com contratura voluntária, fígado palpável a cerca de $3 \mathrm{~cm}$ do rebordo costal direito, sendo o restante do exame normal. A radiografia de tórax evidenciou velamento em base esquerda com aspecto de derrame pleural e atelectasia e o REED foi sugestivo de eventração diafragmática.

0 paciente foi internado, recebendo suporte nutricional enteral e cobertura antibiótica (ceftriaxona e metronidazol). Uma semana após a admissão, na radiografia de tórax foi constatado aumento acentuado do derrame pleural com importante desvio do mediastino contralateral (Figura 1). Nesse mesmo período, houve o aparecimento de um abaulamento volumoso em região epigástrica, indolor e depressível à palpação.

Nesse período foi realizado ultra-som, que mostrou grande coleção cística ( $>20 \mathrm{~cm}$ ) em andar superior de abdome, sugestivo de pseudocisto de pâncreas, e derrame pleural volumoso à esquerda. Esses achados foram confirmados pela tomografia toracoabdominal realizada na seqüência, que apresentava volumosa coleção hipodensa, arredondada, de contornos bem definidos, circundada por fina cápsula medindo 11,6 x 8,8cm (Figura 2) e descartando a possibilidade de eventração diafragmática.

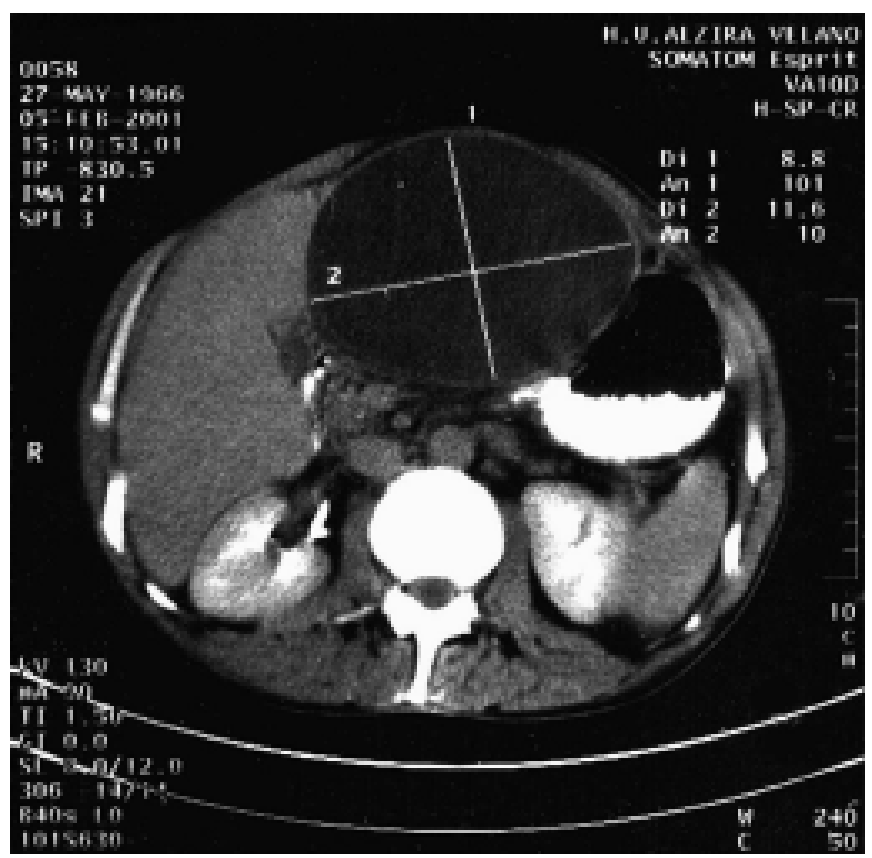

Figura 2 - Tomografia toracoabdominal; F: fígado; $E_{c}$ : estômago contrastado.

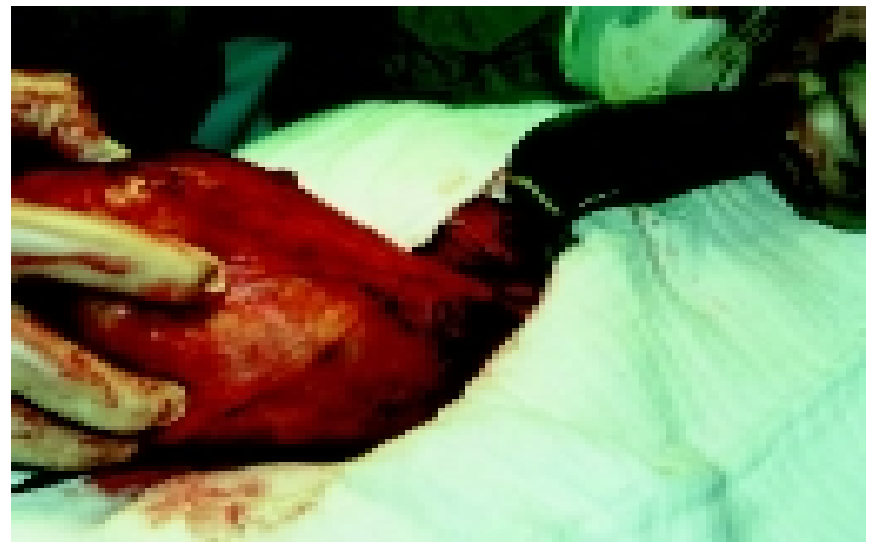

Figura 3 - PC pancreático em região justadiafragmática

O hemograma demonstrou $\mathrm{Hm}=3$ milhões, $\mathrm{Ht}=27 \%$, $\mathrm{Hb}=7,5 \mathrm{~g} \%$; com 7.000 leucócitos ( $69 \%$ de neutrófilos e $26 \%$ de linfócitos). A dosagem da amilase sérica foi de $551 \mathrm{UI} / \mathrm{L}$. O PPD revelou-se negativo, assim como as cinco amostras de escarro para pesquisa de BAAR e fungos.

Foi realizada toracocentese com esvaziamento pleural de aproximadamente $3.200 \mathrm{ml}$, com líquido de coloração acastanhada. A dosagem de proteínas foi de 3,1g/dl e a de glicose, de $79,6 \mathrm{mg} / \mathrm{dl}$. A citometria apresentou presença de leucócitos, contagem diferencial com predomínio de mononucleares ( $60 \%$ de linfócitos) e ausência de malignidade. Pesquisas de BAAR, gram e bacteriológica 
foram negativas; $\mathrm{pH}=7,79$ e $\mathrm{LDH}=405 \mathrm{UI} / \mathrm{ml}$, com amilase de 1.207,0UI/L.

Após esvaziamento pleural, houve melhora clínico-radiológica com centralização do mediastino e diminuição do abaulamento abdominal. Nessas condições, foi repetido o US, que revelou presença de PC com diminuição de volume, mas apresentando paredes espessas e ecos puntiformes em seu interior.

Com estabilização clínica/nutricional, foi indicada laparotomia para tratamento cirúrgico do pseudocisto de pâncreas. Durante 0 ato operatório, foi encontrado PC bem delimitado em andar superior de abdome com pedículo inserido em região esternal diafragmática, sem continuidade com o pâncreas ou qualquer outro órgão. Após ligadura do pedículo, O PC foi retirado totalmente, sem dificuldade (Figura 3). A dosagem de amilase no conteúdo do PC foi de 2.600 unidades UI/L e o exame anatomopatológico foi compatível com pseudocisto peritoneal gigante. 0 paciente apresentou boa evolução no pós-operatório, recebendo alta em boas condições clínicas.

\section{DISCUSSÃO}

Pseudocisto (PC) de pâncreas é uma coleção que contém tecidos, líquido, fragmentos, enzimas pancreáticas e sangue, encerrada por parede não epitelizada de tecido fibroso ou de granulação, que pode desenvolver-se próximo ao ou no interior do órgão ${ }^{(1-3)}$. Trata-se de uma condição relativamente rara, que surge em 10 a $20 \%$ dos pacientes como complicação de pancreatite, aguda ou crônica, ou de trauma pancreático ${ }^{(1,3,5)}$. Aproximadamente, $5 \%$ dos pacientes com PC irão desenvolver derrame pleural, que geralmente ocorre após o episódio agudo da doença ou até mesmo sem nenhuma história prévia de pancreatite. A maioria dos pacientes com derrame pleural decorrente de PC pancreático é do sexo masculino e, em mais de $90 \%$ dos casos, apresenta história de etilismo crônico ${ }^{(3,4,6)}$. Esses derrames tendem a ser recorrentes, são exsudativos, com níveis elevados de amilase no líquido pleural, apresentando coloração acastanhada bastante característica e de grande importância para o diagnóstico de derrames pleurais decorrentes de patologias pancreáticas ${ }^{(2,3,6)}$.

$\mathrm{Na}$ patogênese do derrame pleural decorrente de PC pancreático, o mecanismo mais aceito baseia-se na formação de um trajeto fistuloso entre o pâncreas e a cavidade pleural. A fístula do pâncreas ocorre quando há lesão do ducto pancreático, com conseqüente escoamento de líquido pancreático. Esse líquido inflamatório poderia seguir o trajeto do plexo linfático que conecta a cavidade abdominal à torácica, o qual, além de causar irritação química no plexo, explicaria a relativa predominância dos derrames à esquerda ( $60 \%$ dos casos). De outra forma, menos provável, esse líquido abriria caminho pelo retroperitônio, passando pelo hiato esofágico ou aórtico, e atingiria o mediastino, resultando em mediastinite, a qual poderia estender-se para uma ou ambas as cavidades pleurais. Outras vezes, poderia existir uma comunicação direta do PC com o espaço pleural pelo diafragma ${ }^{(1-4,6)}$.

Nas manifestações clínicas decorrentes da fístula pancreática para o espaço pleural, predominam os sintomas pulmonares, com mais freqüência a dispnéia, tosse e dor torácica. Os sintomas abdominais, de forma geral, têm pouca expressão clínica(1,3-5).

0 diagnóstico de derrame pleural de origem pancreática é relativamente fácil de estabelecer, desde que se encontrem níveis de amilase extraordinariamente altos. 0 diagnóstico diferencial com derrames ricos em amilase e de origem não-pancreática é pequeno e habitualmente com história bem diferenciada, como nas doenças malignas e nas rupturas esofágicas ${ }^{(3,4)}$.

Com exclusão da cirurgia, somente os exames de imagem comprovam o diagnóstico. Os exames de imagem que habitualmente nos permitem estabelecer o diagnóstico são o ultra-som abdominal e/ou a tomografia computadorizada toracoabdominal. Atualmente, dá-se preferência à tomografia pela melhor definição de imagem do PC e das estruturas vizinhas. A colangiopancreatografia retrógrada endoscópica também pode ser usada, tendo sua principal utilização na demonstração do trajeto fistuloso, sendo comumente realizada no pré-operatório, ou ainda no intra-operatório, para melhor programação cirúrgi$\mathrm{ca}^{(1,3,4,7) \text {. }}$

A conduta terapêutica deve ser inicialmente conservadora e pode incluir o repouso do tubo digestivo alcançado através do suporte nutricional parenteral. Pode-se também fazer uso de atropina, análogos da somatostatina, como 0 acetato de octreotide, que aceleram o fechamento da fístula por diminuir a secreção pancreática ${ }^{(3,6)}$. Deve ser realizada toracocentese ou até mesmo drenagem torácica em selo d'água e aspiração contínua, se necessário. É relatado que a eficácia desse tratamento conservador varia de 20 a 50\%, com níveis de recorrência atingindo $15 \%$, o que justificaria uma terapia mais efetiva $a^{(2,3)}$.

0 tratamento cirúrgico para a correção da fístula está na dependência da permanência de alto débito diário do líquido para a cavidade pleural, associado ao insucesso do regime conservador implementado por, pelo menos, duas a três semanas. A cirurgia visa a exérese do segmento pancreático fistuloso. Pode-se, ainda, recorrer à drenagem do PC através de uma fístula externa (percutânea) ou interna (quando há drenagem do PC para o estômago) com pancreatojejunostomia para facilitação da drenagem do canal de Wirsung, com reconstrução intestinal à " $Y$ " de Roux ${ }^{(1,3)}$. 
Pode-se observar que 0 caso relatado apresenta variações quanto ao padrão clássico de apresentação de derrame pleural decorrente de PC. Devido aos achados cirúrgicos, a explicação mais razoável para o desenvolvimento desse derrame pleural está vinculada à extensão do PC

\section{REFERÊNCIAS}

1. Mello LIL, Elabras Filho J, Domingues G, Paschoal ME. Fístula pancreatopleural direita. JBM 1995;69:109-10.

2. Closset J, Gelin M. The management of pancreatic ascites and pancreatic-pleural effusion. Acta Gastroenterol Belg 2000;63:269.

3. Light RW. Pleural effusion secondary to diseases of the gastrointestinal tract. In: Light RW, editor. Pleural diseases. $3^{\text {th }}$ ed. Baltimore: Williams \& Wilkins, 1995;192-207. por trajeto direto, com o espaço pleural passando pelo diafragma. A pós a toracocentese, com a melhora do derrame pleural, e de acordo com os achados do ultra-som e o aumento subseqüente do PC, foi indicada laparotomia.

4. Carvalho CM, Fernandes MG, Abrantes PF, Costa AFC. Fístula pancreatopleural - Relato de caso. J Pneumol 1990;16:237-41.

5. Rose EA, Munawar H, Yang SK, Telmos AJT. Mediastinal extension of a pancreatic pseudocyst. Am J Gastroenterol 2000;95:3638-9.

6. Takeo C, Myojo S. Market effect of octreotide acetate in a case of pancreatic pleural effusion Curr Med Res Opin 2000;16:171-7.

7. Lemay K, Gray R, Numerow L. Answer to case of the month \#79 mediastinal pancreatic pseudocyst. Can Assoc Radiol J 2000;51:358-60. 